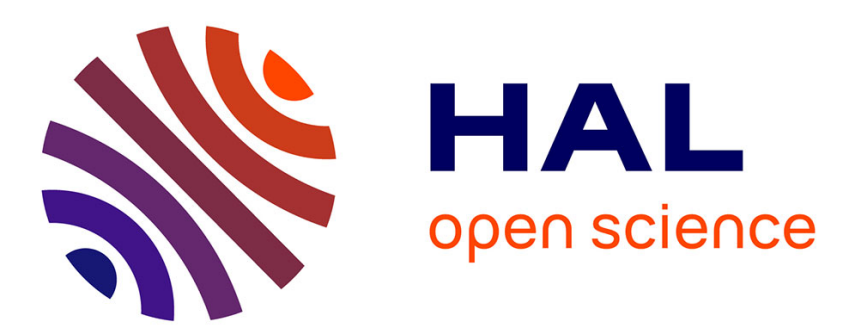

\title{
On a strategy for the reduction of the lack of knowledge (LOK) in model validation
}

Pierre Ladevèze, Guillaume Puel, Thierry Romeuf

\section{To cite this version:}

Pierre Ladevèze, Guillaume Puel, Thierry Romeuf. On a strategy for the reduction of the lack of knowledge (LOK) in model validation. Reliability Engineering and System Safety, 2006, 91 (10-11), pp.1452-1460. 10.1016/j.ress.2005.11.058 . hal-00258642

\section{HAL Id: hal-00258642 \\ https://hal.science/hal-00258642}

Submitted on 22 Feb 2008

HAL is a multi-disciplinary open access archive for the deposit and dissemination of scientific research documents, whether they are published or not. The documents may come from teaching and research institutions in France or abroad, or from public or private research centers.
L'archive ouverte pluridisciplinaire HAL, est destinée au dépôt et à la diffusion de documents scientifiques de niveau recherche, publiés ou non, émanant des établissements d'enseignement et de recherche français ou étrangers, des laboratoires publics ou privés. 


\title{
On a Strategy of the Reduction of the Lack Of Knowledge (LOK) in Model Validation
}

\author{
P. Ladevèze ${ }^{a, *}$, G. Puel ${ }^{a}$, T. Romeuf ${ }^{b}$ \\ ${ }^{\mathrm{a}}$ LMT-Cachan (ENS Cachan/CNRS/Paris 6 University) \\ 61 avenue du Président Wilson 94235 Cachan Cedex France \\ ${ }^{\mathrm{b}}$ European Aeronautic Defence and Space Company (EADS Space Transportation) \\ Route de Verneuil BP 9678133 Les Mureaux Cedex France
}

\begin{abstract}
The quantification of the quality of a structural dynamical model remains a major issue today, and increasingly numerous methods are being devised in order to validate a model by comparison with an experimental reference. This paper presents a theory based on the concept of Lack Of Knowledge, which consists in globalizing the various sources of errors on the substructure level using a scalar internal variable, called the LOK variable, defined over an interval whose upper and lower bounds follow probabilistic laws. These intervals defined on the different substructures are then rigorously propagated through the mechanical model in order to determine intervals with stochastic bounds including a given quantity of interest defined on the whole structure. A general strategy of reduction of the lack of knowledge is then discussed and applied to academic examples as well as industrial cases.
\end{abstract}

Key words: lack of knowledge, uncertainty, model validation, structural dynamics

\section{Introduction}

The quantification of the quality of a structural dynamical model remains a major issue today; with regard to the comparison with an experimental reference, numerous methods have been developed, as in [1] or [2], in order to adjust the stiffness and mass properties of dynamic models based on free- or

\footnotetext{
* Corresponding author.

Email addresses: pierre.ladeveze@lmt.ens-cachan.fr (P. Ladevèze), guillaume.puel@lmt.ens-cachan.fr (G. Puel), thierry.romeuf@space.eads.net (T. Romeuf).
} 
forced-vibration tests. However, even an adjusted model is unable to account for certain phenomena properly: there can be dispersion in the material properties; or the representation of some features, such as the connections, can be oversimplified.

In order to describe such uncertainties, probabilistic methods are being increasingly used [3]; in general, these methods consist in studying the consequences of the uncertainties in the model's parameters on the variability of the results. But one can also find methods which do not necessarily involve probability laws and are able to deal with uncertainties not related to the pure variability of a given parameter $[4-6]$.

The concept of Lack Of Knowledge (LOK), first introduced in [7], consists in globalizing the various sources of errors on the substructure level using a scalar internal variable, called the LOK variable, defined over an interval whose upper and lower bounds follow probabilistic laws. The pair of stochastic bounds defined on each substructure is called basic LOK; for the time being, only the sources of errors concerning stiffnesses are considered. From these basic LOKs associated to the different substructures, we can compute for the whole structure the effective LOK for a quantity of interest $\alpha$, resulting in an interval with stochastic bounds that we can compare with experimental values; this is achieved by the rigorous propagation of the basic LOK of each substructure and its associated probability law through the mechanical model.

The comparison with experimental reality can then be made through these effective LOKs, aiming to build a method in order to determine the basic LOKs the most representative of the experimental reality. Here, we present the first results, on both academic and industrial applications, of a strategy of using additional experimental information to reduce the LOK [8].

\section{Basic lack of knowledge}

The theory considers the case of a structure belonging to a family of similar, but non necessarily identical, structures: each one can be modeled as an assembly of substructures in which the connections can be viewed as special substructures. It is on the level of these substructures that we choose to quantify the uncertainties, thus globalizing all existing sources of errors. For the time being, let us assume that these sources of errors are of the "structural stiffness" type. With the LOK concept we can easily estimate the comparison between the model and reality, where reality is seen as a family of similar, actual structures, too. 


\subsection{Definition}

The theory is based on the use of a given theoretical, deterministic model which is completed by a "LOK model"; this latter consists in associating each substructure $E$ of an actual structure with a LOK variable $m$ defined over an interval whose bounds $m_{\mathrm{E}}^{+}$and $m_{\mathrm{E}}^{-}$are formally defined as follows:

$$
\left(1-m_{E}^{-}\right) \bar{K}_{E} \leq K_{E} \leq\left(1+m_{E}^{+}\right) \bar{K}_{E}
$$

where $K_{E}$ and $\bar{K}_{E}$ designate the stiffness matrices associated with substructure $E$ for the actual structure and for the deterministic theoretical model respectively; in this formal expression, inequalities can be considered as being applied to the eigenvalues associated to the above matrices.

In practice, to express this inequality, one uses the strain energies:

$$
\left(1-m_{E}^{-}\right) \bar{e}_{E} \leq e_{E} \leq\left(1+m_{E}^{+}\right) \bar{e}_{E}
$$

where $e_{\mathrm{E}}=\frac{1}{2} \underline{U^{T}} K_{E} \underline{U}$ denotes the strain energy of an actual structure belonging to the family of similar structures being studied, and $\bar{e}_{\mathrm{E}}=\frac{1}{2} \underline{U}^{T} \bar{K}_{E} \underline{U}$ denotes the strain energy of the deterministic theoretical model. This inequality must be verified for any displacement field $\underline{U}$. The two quantities $m_{\mathrm{E}}^{+}$and $m_{\mathrm{E}}^{-}$are scalar internal variables with respect to Substructure $E$ which we call upper basic LOK and lower basic LOK respectively.

For each substructure, the LOK $m$ lies within the interval $\left[-m_{\mathrm{E}}^{-} ; m_{\mathrm{E}}^{+}\right]$, and one cannot be more precise than that. This description corresponds to interval analysis $[9,10]$, which can model uncertainties which do not come from a direct variability of the phenomena described. However, without any precaution, this kind of description can lead to overestimated results when propagating the intervals through the model.

That's why we decided to defined the bounds of this interval as stochastic variables characterized by probabilistic laws whose nature is defined a priori and whose characteristics are described by two parameters $\bar{m}_{\mathrm{E}}^{+}$and $\bar{m}_{\mathrm{E}}^{-}$bounding all possible instances of $m_{\mathrm{E}}^{+}$and $m_{\mathrm{E}}^{-}$:

- for example, the probability law chosen can be uniform, and all values of $m_{\mathrm{E}}^{+}$and $m_{\mathrm{E}}^{-}$within $\left[-\bar{m}_{\mathrm{E}}^{-} ; \bar{m}_{\mathrm{E}}^{+}\right]$can occur in the same way;

- of course, in some cases where the model is imperfect (e.g. a nonlinear connection represented by a linear model), the lack of knowledge is such that the basic LOKs are not assigned a probability law: one then decides to merely state that the LOK $m$ lies somewhere within an interval $\left[-\bar{m}_{\mathrm{E}}^{-} ; \bar{m}_{\mathrm{E}}^{+}\right]$, which is now deterministic. In this case, we come back to the simple interval analysis' description, as seen above. 
Other situations can be envisaged, such as the use of a Gaussian probability law which is detailed in the following section.

In the general case, we have just introduced a description that is actually close to approaches deriving from the concept of Imprecise Probabilities [6], without our being aware of this connection at first, which explains the fact we introduced our own tools to deal with the Lacks of Knowledge; however, we will try to make a link between the two approaches in Section 2.4.

\subsection{Illustration}

When the only sources of errors being considered are related to the material, one can reasonably assume that the LOK $m$ of Substructure $E$ follows a centered normal law with the probability density $p(m)$ :

$$
m \in\left[-m_{\mathrm{E}}^{-} ; m_{\mathrm{E}}^{+}\right] \text {with } p(m)=\frac{1}{\sqrt{2 \pi \sigma^{2}}} e^{-\frac{m^{2}}{2 \sigma^{2}}}
$$

The standard deviation $\sigma$ of the Gaussian law can be defined using the quantities $\bar{m}_{\mathrm{E}}^{+}$and $\bar{m}_{\mathrm{E}}^{-}$, for example by setting $\int_{-\bar{m}_{\mathrm{E}}^{-}}^{\bar{m}_{\mathrm{E}}^{+}} p(m) \mathrm{d} m=0.99$; in order to eliminate non-physical samples $\left(-m_{\mathrm{E}}^{-}<-1\right)$, one chooses to prescribe a zero probability density outside $\left[-\bar{m}_{\mathrm{E}}^{-} ; \bar{m}_{\mathrm{E}}^{+}\right]$, even if this means renormalizing it afterwards.

With such a law, the probability of observing $m$ in a given interval $\left[-m_{\mathrm{E}}^{-} ; m_{\mathrm{E}}^{+}\right]$ is:

$$
P\left(-m_{\mathrm{E}}^{-} \leq m \leq m_{\mathrm{E}}^{+}\right)=\int_{-m_{\mathrm{E}}^{-}}^{m_{\mathrm{E}}^{+}} p(m) \mathrm{d} m
$$

Since in (2) the basic LOKs $m_{\mathrm{E}}^{+}$and $m_{\mathrm{E}}^{-}$are defined on both sides of the theoretical model, the previous situation can be described by two independent events:

- either $m \in\left[0 ; m_{\mathrm{E}}^{+}\right]$, i.e. one has the event $\left(m_{\mathrm{E}}^{-}=0, m_{\mathrm{E}}^{+} \geq 0\right)$ with probability $P^{+}\left(m_{\mathrm{E}}^{+}\right)$;

- or $m \in\left[-m_{\mathrm{E}}^{-} ; 0\right]$, i.e. one has the event $\left(m_{\mathrm{E}}^{-} \geq 0, m_{\mathrm{E}}^{+}=0\right)$ with probability $P^{-}\left(m_{\mathrm{E}}^{-}\right)$.

Then, one has $P^{+}(\infty)+P^{-}(\infty)=1$, and even $P^{+}(\infty)=P^{-}(\infty)=\frac{1}{2}$ in the particular case of a centered normal law: This situation is depicted in Figure 1. This case shows how one can represent an uncertainty defined by a classical probability law using our concept of lack of knowledge: depending on the value of $m$ obtained by random drawing, one has two distinct types of intervals: $\left[0 ; m_{\mathrm{E}}^{+}\right]$and $\left[-m_{\mathrm{E}}^{-} ; 0\right]$. 


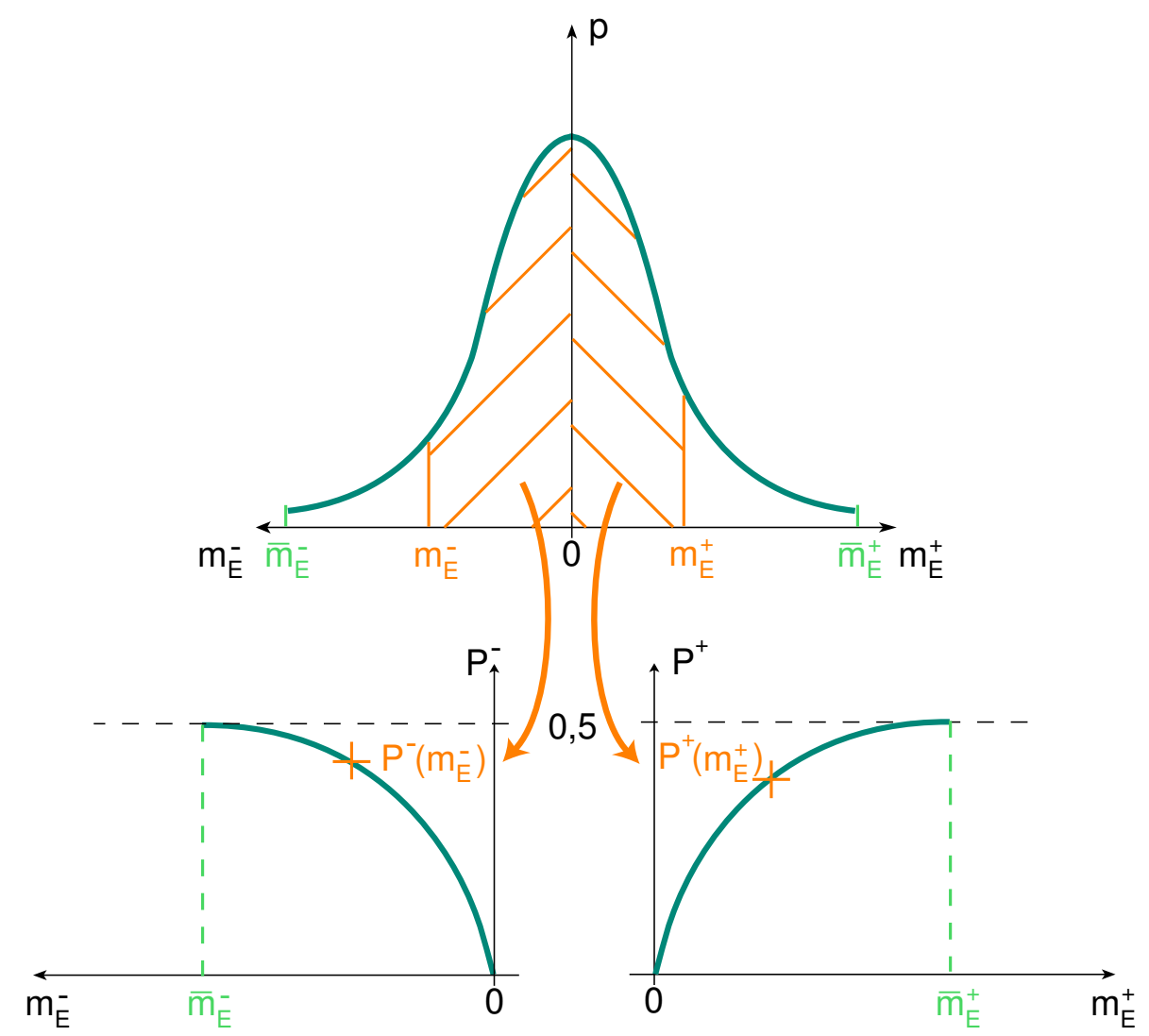

Fig. 1. Case of a Gaussian probability law (up) probability density of $m$ (down) $P^{+}$and $P^{-}$

Of course, other probability laws could be assumed: the important idea is that, with the use of the quantities $P^{-}(\infty)+P^{+}\left(m_{\mathrm{E}}^{+}\right)$and $P^{+}(\infty)+P^{-}\left(m_{\mathrm{E}}^{-}\right)$as respective distribution functions of the upper bound $m_{\mathrm{E}}^{+}$and the lower bound $m_{\mathrm{E}}^{-}$, we can deal with situations where the direct use of a probability density $p(m)$ might not be applied.

\subsection{The concept of LOK-probability}

However, the use of two quantities $P^{+}$and $P^{-}$is, in the general case, relatively complex, so a number of mathematical, pragmatical tools were introduced in $[11]$.

Let us consider a family of intervals $\left[-m_{\mathrm{E}}^{-} ; m_{\mathrm{E}}^{+}\right] \ni m$ with $m_{\mathrm{E}}^{+}+m_{\mathrm{E}}^{-}=L$. For a given $L$, the interval $I(L)=\left[-m_{\mathrm{E}}^{-} ; m_{\mathrm{E}}^{+}\right]$is said to be standard if the probability of having $m$ in $I(L)$ is maximum over the set of all intervals with 
length $L$, which we can write mathematically as:

$$
I(L)=\arg \max _{\substack{\left[-m_{\mathrm{E}}^{-} ; m_{\mathrm{E}}^{+}\right] \\ m_{\mathrm{E}}^{+}+m_{\mathrm{E}}^{-}=L}} P^{+}\left(m_{\mathrm{E}}^{+}\right)+P^{-}\left(m_{\mathrm{E}}^{-}\right)
$$

With this definition, one can introduce the concept of $L O K$ - probability $P(L)$ by stating that for a given $L, P(L)$ is the probability associated to the definition $(5)$ of $I(L)$ :

$$
P(L)=\max _{\substack{\left[-m_{\mathrm{E}}^{-} ; m_{\mathrm{E}}^{+}\right] \\ m_{\mathrm{E}}^{+}+m_{\mathrm{E}}^{-}=L}} P^{+}\left(m_{\mathrm{E}}^{+}\right)+P^{-}\left(m_{\mathrm{E}}^{-}\right)
$$

Thus, as $P^{+}\left(m_{\mathrm{E}}^{+}\right)$and $P^{-}\left(m_{\mathrm{E}}^{-}\right)$are linked to the distribution function of the occurrences of the upper bound $m_{\mathrm{E}}^{+}$and the lower bound $m_{\mathrm{E}}^{-}$respectively, the uncertain variable $m$ has a probability bigger than $P^{+}\left(m_{\mathrm{E}}^{+}\right)+P^{-}\left(m_{\mathrm{E}}^{-}\right)$ being within $\left[-m_{\mathrm{E}}^{-} ; m_{\mathrm{E}}^{+}\right]$: indeed, $P^{+}\left(m_{\mathrm{E}}^{+}\right)+P^{-}\left(m_{\mathrm{E}}^{-}\right)<1$ means that intervals including $\left[-m_{\mathrm{E}}^{-} ; m_{\mathrm{E}}^{+}\right]$could be sampled, which increases the probability of $m$ being within $\left[-m_{\mathrm{E}}^{-} ; m_{\mathrm{E}}^{+}\right]$.

Another possible interpretation of these definitions is that if one seeks an interval of basic LOKs such that $m$ has at least a given probability $P$ of being inside, one has to consider the standard interval $I\left(L_{\mathrm{P}}\right)$ such that the associated LOK-probability $P(L)$ equals $P$. One can show that this interval $I\left(L_{\mathrm{P}}\right)$ is in fact the smallest interval $\left[-m_{\mathrm{E}}^{-} ; m_{\mathrm{E}}^{+}\right]$such that $P^{+}\left(m_{\mathrm{E}}^{+}\right)+P^{-}\left(m_{\mathrm{E}}^{-}\right)=P$, which we can write mathematically as:

$$
I\left(L_{\mathrm{P}}\right)=\arg \min _{\substack{\left[-m_{\mathrm{E}}^{-} ; m_{\mathrm{E}}^{+}\right] \\ P^{+}\left(m_{\mathrm{E}}^{+}\right)+P^{-}\left(m_{\mathrm{E}}^{-}\right)=P}} m_{\mathrm{E}}^{+}+m_{\mathrm{E}}^{-}
$$

The situation is depicted in Figure 2, where we show the standard interval from a family of intervals of same length $L$.

In the case of uniform laws, there is not unicity, but what matters is to be able to consider a family of standard intervals defined by only one stochastic variable, which simplifies the analysis.

\subsection{Comparison with the Dempster-Shafer sets}

As we outlined it before, the way we defined the basic LOKs can be related to the concepts of Imprecise Probabilities [6]. In this section, we try to set a bridge with the Dempster-Shafer sets $[12,13]$, which are particular cases of the theory of Imprecise Probabilities. In order to simplify the comparison, we 


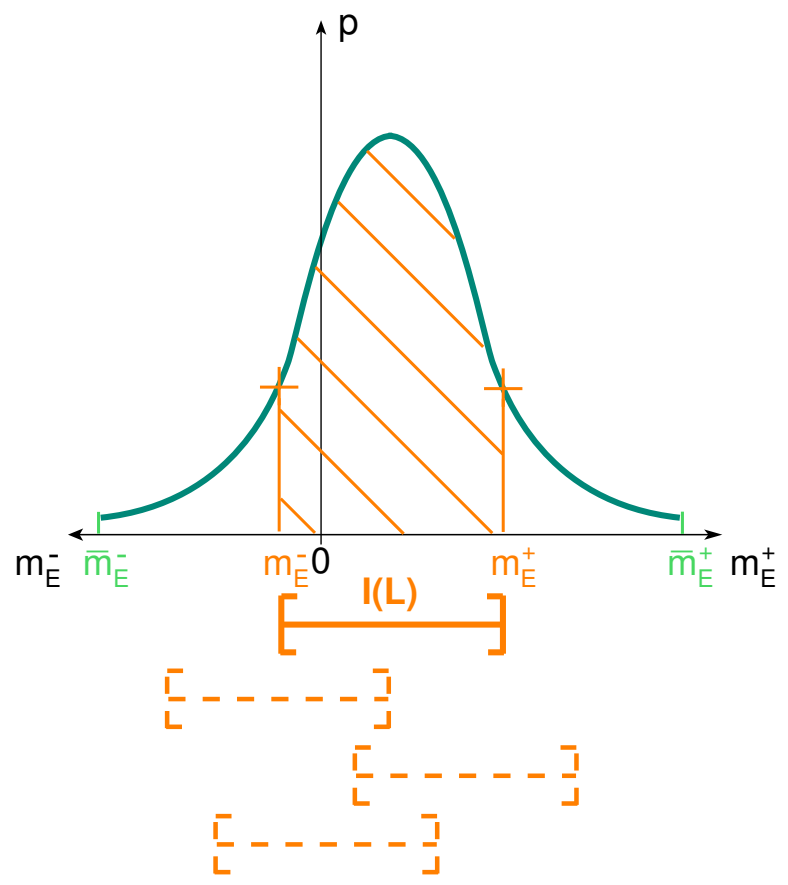

Fig. 2. Standard interval of length $L$

only consider here intervals of the kind $\left[0 ; m_{\mathrm{E}}^{+}\right]$, with associated probabilities $P^{+}\left(m_{\mathrm{E}}^{+}\right)$.

With 4 points of discretization for example, such that:

$$
P^{+}\left(m_{i}^{+}\right)=\frac{i}{4} \quad \forall i=1, \ldots, 4
$$

we can define a Dempster-Shafer set $\mathcal{I}$ characterized by the following focal elements $A_{i}$ and probabilistic assignments $m\left(I_{i}\right)$ :

$$
I_{i}=\left[0 ; m_{i}^{+}\right] \quad \text { and } \quad m\left(I_{i}\right)=\frac{1}{4} \quad \forall i=1, \ldots, 4
$$

The probability for $m$ being within an interval $J \subset \mathbb{R}^{+}$is given by the Dempster rule of combination [12]:

$$
\sum_{I_{i} \subset J} m\left(I_{i}\right) \leq P(J) \leq \sum_{I_{i} \cap J \neq \emptyset} m\left(I_{i}\right)
$$

With the particular case $J=I_{k}$, where $k$ is given, we get:

$$
\frac{k}{4}=\sum_{I_{i} \subset I_{k}} m\left(I_{i}\right) \leq P\left(I_{k}\right) \leq \sum_{I_{i} \cap I_{k} \neq \emptyset} m\left(I_{i}\right)=1
$$

The previous relation means that the probability for $m$ being within $I_{k}$ is at least $\frac{k}{4}=P^{+}\left(m_{k}^{+}\right)$. This is coherent with our previous assertion, that 
$P^{+}\left(m_{\mathrm{E}}^{+}\right)+P^{-}\left(m_{\mathrm{E}}^{-}\right)$is the best minor value of the probability for $m$ being the interval $\left[-m_{\mathrm{E}}^{-} ; m_{\mathrm{E}}^{+}\right]$.

In the following sections, we will use the LOK-probability in order to describe the effect of the basic LOKs on quantities of interest defined on the whole structure.

\section{Utilization of the lacks of knowledge}

\subsection{Principle}

Let us consider a quantity of interest $\alpha$ relative to the whole structure $\Omega$ from the family of similar structures considered. We define $\Delta \alpha_{\bmod }=\alpha_{\bmod }-\bar{\alpha}$, where $\alpha_{\text {mod }}$ is the quantity of interest associated with the model using the LOK concept; $\bar{\alpha}$ is the value associated with the deterministic theoretical model.

Regarding the LOK model, one can associate each instance of $\left(m_{\mathrm{E}}^{-}, m_{\mathrm{E}}^{+}\right)_{E \in \Omega}$ with two bounds $\boldsymbol{\Delta} \boldsymbol{\alpha}_{\text {mod }}^{+}$and $\boldsymbol{\Delta} \boldsymbol{\alpha}_{\text {mod }}^{-}$of the quantity $\Delta \alpha_{\text {mod }}$ : as we will see in Section 3.2, this is simply a matter of propagating the basic LOK intervals $\left(\left[-m_{\mathrm{E}}^{-} ; m_{\mathrm{E}}^{+}\right]\right)_{E \in \Omega}$. As long as one knows the probability laws of the basic LOKs, one can determine the distribution of these bounds $\boldsymbol{\Delta} \boldsymbol{\alpha}_{\bmod }^{+}$and $\boldsymbol{\Delta} \boldsymbol{\alpha}_{\mathbf{m o d}}^{-}$in the form of an LOK-probability $P_{\alpha}(L)$ such that:

$$
P\left(\Delta \alpha_{\text {mod }} \in I_{\Delta \alpha_{\text {mod }}}(L)\right)=P_{\alpha}(L) \forall L
$$

Again, this is the same thing as considering for a given probability $P$ the standard interval $I_{\Delta \alpha_{\text {mod }}}\left(L_{\mathrm{P}}\right)$ such that $P\left(\Delta \alpha_{\text {mod }} \in I_{\Delta \alpha_{\text {mod }}}\left(L_{\mathrm{P}}\right)\right)$ is bigger than $P$. The two bounds of this interval $I_{\Delta \alpha_{\text {mod }}}(L \mathrm{P})$, denoted $\Delta \alpha_{\text {mod }}^{-}(P)$ and $\Delta \alpha_{\text {mod }}^{+}(P)$, constitute what we call the effective $L O K$ on the quantity of interest $\alpha$. So we are able to derive accurate information about the quantity of interest $\alpha_{\text {mod }}$.

\subsection{Effective LOKs}

The calculation of effective LOKs applies to quantities of interest which are common in modal analysis. Here, we are interested in measurements of free vibrations; therefore, we are using eigenpulsations $\omega_{i}$ and eigenmodes $\underline{\phi}_{i}$ as the quantities of interest $\alpha$ : they are defined by the following eigenvalues problem:

$$
\left(K-\omega_{i}^{2} M\right) \underline{\phi}_{i}=\underline{0}
$$


where $K$ and $M$ are the global stiffness and mass matrices respectively; moreover, the global stiffness matrix can be split on the different substructures $E$ of the structure $\Omega: K=\sum_{E \in \Omega} K_{E}$.

Let us recall that the pair $\Delta \alpha_{\text {mod }}^{-}, \Delta \alpha_{\text {mod }}^{+}$constitutes what we call the effective $L O K$ related to the quantity $\Delta \alpha_{\text {mod }}$; the determination of these two quantities for eigenpulsations and eigenmodes is given in the following two sections. It relies on modal analysis classical relations of eigenmodes' variations.

\subsubsection{Effective LOK on an eigenpulsation}

If the modes $\bar{\phi}_{i}$ of the deterministic theoretical model are normalized with respect to the mass matrix $\left(\left(\underline{\phi}_{j}^{T} \overline{\mathbf{M}}_{i}=\delta_{j i}\right)\right.$, we can express the discrepancy between $\omega_{i}^{2}$ and $\bar{\omega}_{i}^{2}$ as:

$$
\omega_{i}^{2}-\bar{\omega}_{i}^{2}=\underline{\phi}_{i}^{T} K \underline{\phi}_{i}-\underline{\phi}_{i}^{T} \bar{K}_{i}
$$

A first-order approximation yields then:

$$
\omega_{i}^{2}-\bar{\omega}_{i}^{2} \simeq \underline{\bar{\phi}}_{i}^{T}(K-\bar{K}) \underline{\phi}_{i}=2 \sum_{E \in \Omega}\left(e_{E}\left(\underline{\bar{\phi}}_{i}\right)-\bar{e}_{\mathrm{E}}\left(\underline{\bar{\phi}}_{i}\right)\right)
$$

by definition of the strain energies.

With the previous equation, the fundamental relation (2) enables us to propagate the intervals $\left(\left[-m_{\mathrm{E}}^{-} ; m_{\mathrm{E}}^{+}\right]\right)_{E \in \Omega}$ (where, for each substructure $E,\left(m_{\mathrm{E}}^{-}, m_{\mathrm{E}}^{+}\right)$ is a given sample of the basic LOKs according to the probability laws chosen) in a rigorous way as follows:

$$
-\Delta \omega_{i \bmod }^{2-} \leq \omega_{i}^{2} \leq \Delta \omega_{i \bmod }^{2+}
$$

with

$$
\begin{aligned}
& \boldsymbol{\Delta} \boldsymbol{\omega}_{\boldsymbol{i} \text { mod }}^{\mathbf{2}}=2 \sum_{E \in \Omega} m_{\mathrm{E}}^{-} \bar{e}_{\mathrm{E}}\left(\underline{\phi}_{i}\right) \\
& \boldsymbol{\Delta} \boldsymbol{\omega}_{\boldsymbol{i} \text { mod }}^{\mathbf{2}+}=2 \sum_{E \in \Omega} m_{\mathrm{E}}^{+} \bar{e}_{\mathrm{E}}\left(\bar{\phi}_{i}\right)
\end{aligned}
$$

If one knows the probability laws for the basic LOKs, one can obtain the dispersions of the bounds $\Delta \boldsymbol{\omega}_{\boldsymbol{i} \text { mod }}^{2-}$ and $\boldsymbol{\Delta} \boldsymbol{\omega}_{\boldsymbol{i} \text { mod }}^{2+}$ and, thus, determine for a given probability $P$ the two bounds $\Delta \omega_{i \text { mod }}^{2-}$ and $\Delta \omega_{i \bmod }^{2+}$ of the associated standard interval $I_{\Delta \omega_{i \text { mod }}^{2}}\left(L_{\mathrm{P}}\right)$; in other words, one obtains the effective LOK on the square of the eigenpulsation $\omega_{i}^{2}$. 


\subsubsection{Effective $L O K$ on an eigenmode}

As the modes $\underline{\phi}_{i}$ are not scalar quantities, one is interested with their values $\underline{\phi}_{k i}$ at a specific degree of freedom (DOF) $k$, or else with their projection $\underline{\phi}_{i}^{\mathrm{N}}$ according to a given direction $\underline{N}$. In both cases, for small samples $\left(m_{\mathrm{E}}^{-}, m_{\mathrm{E}}^{+}\right)_{E \in \Omega}$, one can express the dispersions of the bounds $\Delta \phi_{\boldsymbol{k i ~ m o d}}^{-}$and $\Delta \phi_{\boldsymbol{k} \text { mod }}^{+}$, and of $\boldsymbol{\Delta} \phi_{\boldsymbol{i} \text { mod }}^{\mathbf{N}-}$ and $\boldsymbol{\Delta} \phi_{\boldsymbol{i} \text { mod }}^{\mathbf{N}+}$ respectively, as linear combinations of the basic LOKs: the resulting relations come from the classical Nelson's formula [14]. For example, we get the following relations for the calculation of the bounds $\Delta \phi_{i \text { mod }}^{\mathbf{N}-}$ and $\Delta \phi_{i \text { mod }}^{\mathbf{N}+}$ :

$$
-\Delta \boldsymbol{\phi}_{\boldsymbol{i} \bmod }^{\mathbf{N}-} \leq\left(\underline{\phi}_{i}-\underline{\phi}_{i}\right) \cdot \underline{N} \leq \boldsymbol{\Delta} \boldsymbol{\phi}_{\boldsymbol{i} \text { mod }}^{\mathbf{N}+}
$$

with

$$
\begin{aligned}
& \Delta \phi_{\boldsymbol{i} \text { mod }}^{\mathbf{N}-}=\sum_{E \in \Omega} \sum_{\substack{k=1 \\
k \neq i}}^{r} \frac{1}{2\left(\bar{\omega}_{k}^{2}-\bar{\omega}_{i}^{2}\right)}\left\{\boldsymbol{m}_{\mathbf{E}}^{-} \bar{e}_{\mathrm{E}}\left(\underline{\phi}_{i}+\underline{\phi}_{k}\right)+\boldsymbol{m}_{\mathbf{E}}^{+} \bar{e}_{\mathrm{E}}\left(\underline{\phi}_{i}-\underline{\phi}_{k}\right)\right\} \underline{\phi}_{k} \cdot \underline{N} \\
& \Delta \phi_{\boldsymbol{i} \text { mod }}^{\mathbf{N}+}=\sum_{E \in \Omega} \sum_{\substack{k=1 \\
k \neq i}}^{r} \frac{1}{2\left(\bar{\omega}_{k}^{2}-\bar{\omega}_{i}^{2}\right)}\left\{\boldsymbol{m}_{\mathbf{E}}^{+} \bar{e}_{\mathrm{E}}\left(\bar{\phi}_{i}+\underline{\phi}_{k}\right)+\boldsymbol{m}_{\mathbf{E}}^{-} \bar{e}_{\mathrm{E}}\left(\bar{\phi}_{i}-\underline{\phi}_{k}\right)\right\} \underline{\phi}_{k} \cdot \underline{N}
\end{aligned}
$$

Then, for a given probability $P$, one determines the two bounds $\Delta \phi_{i \text { mod }}^{\mathrm{N}-}$ and $\Delta \phi_{i \text { mod }}^{\mathrm{N}+}$ of the associated standard interval $I_{\Delta \phi_{i \bmod }^{\mathrm{N}}}\left(L_{\mathrm{P}}\right)$; in other words, one obtains the effective LOK on the value of the projection $\phi_{i} \cdot \underline{N}$. More details can be found in [15].

\subsubsection{Effective LOKs and sensitivity analysis}

In a certain way, the previous equations can be compared with the goals of the global sensitivity analysis [16], which studies how the uncertainty in the output of a model can be apportioned to different sources of uncertainty in the model input.

Indeed, the different relations we established between the basic LOKs and the bounds including the chosen quantity of interest, show that the latter can be expressed as linear combinations of the basic LOKs of the different substructures, quantifying by this way how the uncertainty on each substructure applies to the total uncertainty on the quantity of interest: we can write formally for the general case: 


$$
\begin{aligned}
& \boldsymbol{\Delta} \boldsymbol{\alpha}_{\mathbf{m o d}}^{-}=\sum_{E \in \Omega} \Delta \alpha_{E}^{-}=\sum_{E \in \Omega}\left(a_{E}^{-} \boldsymbol{m}_{\mathbf{E}}^{-}+b_{E}^{-} \boldsymbol{m}_{\mathbf{E}}^{+}\right) \\
& \boldsymbol{\Delta} \boldsymbol{\alpha}_{\mathbf{m o d}}^{+}=\sum_{E \in \Omega} \Delta \alpha_{E}^{+}=\sum_{E \in \Omega}\left(a_{E}^{+} \boldsymbol{m}_{\mathbf{E}}^{-}+b_{E}^{+} \boldsymbol{m}_{\mathbf{E}}^{+}\right)
\end{aligned}
$$

These relations can then lead to a strategy of selection of the most relevant experimental tests in order to determine the basic LOKs the most representative of the experimental dispersion, as presented in the following section.

\subsection{Determination of the basic LOKs}

\subsubsection{Comparison with an experimental reference}

The quantification of the quality of a model with respect to an experimental reference using our theory involves the determination of the basic LOKs which best characterize the dispersions observed. Regarding the family of actual structures, one can determine two values $\Delta \alpha_{\exp }^{-}$and $\Delta \alpha_{\exp }^{+}$which, for a given probability $P$, contain $P \%$ of the values of the quantity of interest $\Delta \alpha_{\exp }$ related to the actual structures.

Then, using the concept of LOK-probability, the comparison of the experimental data with the values given by the LOK model can be made by saying that the basic LOKs must be such that:

$$
P\left(\Delta \alpha_{\exp } \in I_{\Delta \alpha_{\text {mod }}}(L)\right) \geq P_{\alpha}(L) \forall L
$$

Through this relation, we claim that the LOK-model should be determined on the safe side, that is the LOK-probability, which is a lower bound of the real probability for $\Delta \alpha_{\text {mod }}$ being in the standard interval $I_{\Delta \alpha_{\text {mod }}}\left(L_{\mathrm{P}}\right)$ should be a lower bound of the probability for the experimental quantity of interest $\Delta \alpha_{\exp }$ being within $I_{\Delta \alpha_{\text {mod }}}\left(L_{\mathrm{P}}\right)$.

This necessary condition is the same as saying, in terms of standard intervals, that the relation $\Delta \alpha_{\text {mod }}^{-}(P) \leq \Delta \alpha_{\text {exp }}^{-}(P) \leq \Delta \alpha_{\text {exp }}^{+}(P) \leq \Delta \alpha_{\text {mod }}^{+}(P)$ must be verified for any given probability value $P$. This interpretation is, in fact, a generalization of the $99 \%$ values mentioned in $[7,15]$.

\subsubsection{Reduction of the basic LOKs}

The main idea behind this determination is that the greater the amount of experimental information available, the more likely one is to reduce the basic 
LOKs. This principle requires an initial description, which may be coarse, but necessarily overestimated, of the basic LOKs for each substructure. This can be obtained through a priori knowledge or through experiments relevant to the structure being studied. In the end, one has a set of initial upper bounds $\left(\bar{m}_{\mathrm{E}}^{+0}, \bar{m}_{\mathrm{E}}^{-0}\right)_{E \in \Omega}$ (associated with probability laws) such that all the constraints (21) for the experimental data available, and which can be used to describe the basic LOKs for each substructure.

The reduction process consists in using this additional relevant experimental information to reduce the level of LOK one substructure at a time. Let us consider a given substructure $E^{*}$. The problem is to determine a basic LOK $\left(\boldsymbol{m}_{\mathrm{E}^{*}}^{-}, \boldsymbol{m}_{\mathrm{E}^{*}}^{+}\right)$which is smaller than the initial LOK $\left(\boldsymbol{m}_{\mathrm{E}^{*}}^{-\mathbf{0}}, \boldsymbol{m}_{\mathrm{E}^{*}}^{+\mathbf{0}}\right)$, which in terms of LOK-probabilities is equivalent to the following inequality:

$$
P_{E^{*}}^{0}(L) \leq P_{E^{*}}(L) \quad \forall L
$$

This reduction is carried out under the constraint (21) associated with the experimental information chosen, which in terms of effective LOK on the quantity of interest $\alpha$ can be written as:

$$
\Delta \alpha_{\text {mod }}^{-}(P) \leq \Delta \alpha_{\text {exp }}^{-}(P) \leq \Delta \alpha_{\text {exp }}^{+}(P) \leq \Delta \alpha_{\text {mod }}^{+}(P)
$$

As the reduction is achieved substructure per substructure, the verification of the constraint (21) is not sufficient in order to get results which are realistic for any situation. Indeed, let us recall that the effective LOK derives from the LOK-probability $P_{\alpha}(L)$ which characterizes the distribution of the bounds $\boldsymbol{\Delta} \boldsymbol{\alpha}_{\text {mod }}^{+}$and $\boldsymbol{\Delta} \boldsymbol{\alpha}_{\text {mod }}^{-}$of the quantity of interest $\Delta \alpha_{\text {mod }}$ relative to the model. As seen in Section 3.2, these bounds contain contributions from all the substructures: for a given set of samples $\left(m_{\mathrm{E}}^{-}, m_{\mathrm{E}}^{+}\right)_{E \in \Omega}$, one has:

$$
\begin{aligned}
& \Delta \boldsymbol{\alpha}_{\text {mod }}^{-}=\Delta \alpha_{E^{*}}^{-}+\sum_{E \neq E^{*}} \Delta \alpha_{E}^{-} \\
& \Delta \boldsymbol{\alpha}_{\text {mod }}^{+}=\Delta \alpha_{E^{*}}^{+}+\sum_{E \neq E^{*}} \Delta \alpha_{E}^{+}
\end{aligned}
$$

Thus, the reduction of the LOK of $E^{*}$ may very well be flawed due to grossly overestimated initial LOKs of the other substructures $E \neq E^{*}$. In order to prevent such an occurrence, one should account for the worst possible cases concerning all other substructures, which can be formally written as:

$$
\begin{aligned}
& \Delta \boldsymbol{\alpha}_{\text {mod }}^{- \text {worst }}=\Delta \alpha_{E^{*}}^{-}+\sum_{E \neq E^{*}} \Delta \alpha_{E}^{- \text {worst }} \\
& \Delta \boldsymbol{\alpha}_{\text {mod }}^{+ \text {worst }}=\Delta \alpha_{E^{*}}^{+}+\sum_{E \neq E^{*}} \Delta \alpha_{E}^{+ \text {worst }}
\end{aligned}
$$

For example, concerning the effective LOK of an eigenfrequency, one has to 
deal with:

$$
\begin{aligned}
& \boldsymbol{\Delta} \boldsymbol{\alpha}_{\mathbf{E}}^{- \text {worst }}=-2 \boldsymbol{m}_{\mathbf{E}}^{+} \bar{e}_{\mathrm{E}}\left(\bar{\phi}_{i}\right) \\
& \boldsymbol{\Delta} \boldsymbol{\alpha}_{\mathbf{E}}^{+ \text {worst }}=-2 \boldsymbol{m}_{\mathbf{E}}^{-} \bar{e}_{\mathrm{E}}\left(\underline{\bar{\phi}}_{i}\right)
\end{aligned}
$$

This analysis of the worst cases should be further enriched by introducing a coefficient $\left.\left.\rho_{E^{*}} \in\right] 0 ; 1\right]$ enabling one to quantify to what extent the selected experimental information is representative of the behavior of the structure: this term, which we call coefficient of representativeness, is maximum when the experimental data account perfectly for the global mechanics of the structure. As an illustration, let us take the example of a traction test: experimental data associated with this test are relevant in the case of an isotropic model of substructure, because they give a trustworthy vision of the LOK associated to the substructure. On the contrary, they constitute a very partial vision in the case of an orthotropic model, by giving information in the direction of the traction test only. So the resulting bounds are defined as follows:

$$
\begin{aligned}
& \boldsymbol{\Delta} \boldsymbol{\alpha}_{\text {mod }}^{- \text {worst }}=\rho_{E^{*}} \Delta \alpha_{E^{*}}^{-}+\sum_{E \neq E^{*}} \Delta \alpha_{E}^{- \text {worst }} \\
& \boldsymbol{\Delta}_{\boldsymbol{\alpha}_{\text {mod }}^{+ \text {worst }}}=\rho_{E^{*}} \Delta \alpha_{E^{*}}^{+}+\sum_{E \neq E^{*}} \Delta \alpha_{E}^{+ \text {worst }}
\end{aligned}
$$

The choice of the coefficient $\rho_{E^{*}}$ is strongly linked to the kind of test considered.

Then, one can associate the bounds $\boldsymbol{\Delta} \boldsymbol{\alpha}_{\text {mod }}^{+ \text {worst }}$ and $\boldsymbol{\Delta} \boldsymbol{\alpha}_{\text {mod }}^{- \text {worst }}$ with an LOKprobability $P_{\alpha^{w o r s t}}\left(L_{\mathrm{P}}\right)$ and deduce the two bounds $\Delta \alpha_{\bmod }^{+ \text {worst }}$ and $\Delta \alpha_{\bmod }^{- \text {worst }}$ of the standard interval $I_{\Delta \alpha_{\text {mod }}^{\text {worst }}}(L)$ associated with a given probability $P$. Thus, new constraints must be taken into account:

$$
\Delta \alpha_{\text {mod }}^{- \text {worst }}(P) \leq \Delta \alpha_{\text {exp }}^{-}(P) \leq \Delta \alpha_{\exp }^{+}(P) \leq \Delta \alpha_{\text {mod }}^{+ \text {worst }}(P)
$$

In summary, the problem of reducing the LOK on a given substructure $E^{*}$ consists in finding

$$
\max P_{E^{*}}(L, \bar{m}) \quad \forall L
$$

which verifies the constraints associated with the experimental information chosen. 


\section{Application to a simple case}

\subsection{Definition of the structure}

\subsubsection{Data of the deterministic theoretical model}

The structure being considered is a plane truss; it consists of the six pinjointed bars shown in Figure 3. The bars are assumed to be solicited only in traction-compression and the connections between the structure and the base are assumed to be perfectly rigid. The material characteristics of the theoretical model are given in Table 1.

\subsubsection{Experimental data}

We consider a family of similar trusses whose eigenpulsations and eigenmodes constitute the experimental data which enable the reduction of the basic LOKs. The "experimental" trusses are simulated by introducing into the deterministic theoretical model stiffness dispersions in the material characteristics; these are indicated in Table 1 . One should note we associated to material "X" a uniform stiffness distribution. One can calculate the eigenpulsations and eigenmodes of each of these simulated trusses and thus determine the laws of distribution of the experimental quantities of interest (e.g. Figure 4).

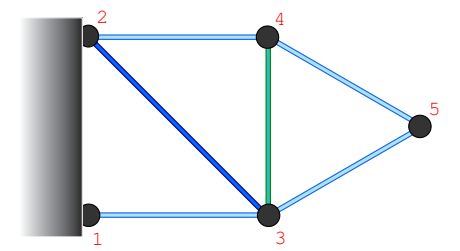

Fig. 3. The plane truss being studied

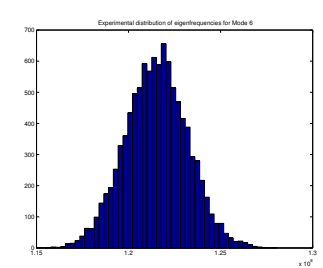

Fig. 4. Experimental distribution of $\omega_{\text {exp }}^{2}$ for Mode 6

Table 1

Material characteristics of the deterministic model of the truss and of the numerically simulated structures, where bars 1-3, 3-5, 4-5 and 2-4 constitute Group g1, bar 2-3 is Group g2 and bar 3-4 is Group 3

\begin{tabular}{|c||c|c|c||c|c|c|}
\hline Gr. & $\begin{array}{c}\text { Mate- } \\
\text {-rial }\end{array}$ & $\begin{array}{c}\text { Young's } \\
\text { modulus }\end{array}$ & $\begin{array}{c}\text { Mass } \\
\text { density }\end{array}$ & $\begin{array}{c}\text { Law } \\
\text { chosen }\end{array}$ & $\begin{array}{c}\text { Relative } \\
\text { domain }\end{array}$ & $\begin{array}{c}\text { Relative characteristics } \\
(\mu \text { : mean } / \sigma \text { : std dev }\end{array}$ \\
\hline g1 & alum. & $\bar{E}_{g 1}=72 G P a$ & $2,700 \mathrm{~kg} / \mathrm{m}^{3}$ & Gaussian & {$[-0.05 ; 0.05]$} & $\mu=0.00 / \sigma=0.019$ \\
g2 & steel & $\bar{E}_{g 2}=210 G P a$ & $7,800 \mathrm{~kg} / \mathrm{m}^{3}$ & Gaussian & {$[-0.15 ; 0.05]$} & $\mu=-0.05 / \sigma=0.039$ \\
g3 & "X" & $\bar{E}_{g 3}=10 G P a$ & $1,500 \mathrm{~kg} / \mathrm{m}^{3}$ & uniform & {$[-0.10 ; 0.20]$} & $\mu=0.10 / \sigma=0.087$ \\
\hline
\end{tabular}




\subsection{Reduction of the basic LOKs}

The reduction process is initiated by prescribing a priori an initial LOK level $\left(\bar{m}_{\mathrm{E}}^{-0}=50 \%, \bar{m}_{\mathrm{E}}^{+0}=50 \%\right)$ on each substructure, which constitutes an appropriate overestimated level. One also assumes that the probability laws of the basic LOKs are Gaussian for the aluminum and steel bars and uniform for the bar made of material "X". The only experimental information retained is the values $\Delta \omega_{i \exp }^{2+}$ and $\Delta \omega_{i \exp }^{2-}$ which bound $99 \%$ of the experimental values of the eigenpulsations actually measured; in particular, one is not concerned with the distribution of these values between these bounds of the standard $99 \%$-probability interval $I_{\Delta \omega_{i \bmod }^{2}}$. If one were to seek a richer description, one could also use other values of probability.

As a second step, it is important to identify the most relevant experimental measurements in order to carry out reductions on the different substructures successfully. An effective method consists in relying upon the fact that the sensitivity of the effective LOKs with respect to the basic LOKs is directly related to the modal strain energies of the deterministic theoretical model, as can be seen in the expressions of Section 3.2. Indeed, the most relevant experimental data in the process of reducing the basic LOKs of the structure $E^{*}$ are those whose modal strain energy is located primarily in $E^{*}$. Since the experimental information we chose concerns eigenpulsations, we need to consider the energies $\bar{e}_{\mathrm{E}}\left(\bar{\phi}_{i}\right)$, which are listed in Table 2 where the most important values are in boldface.

Table 2

Modal strain energies for Modes 1 to 6

\begin{tabular}{|c|c|c|c|c|c|c|}
\hline $\bar{e}_{\mathrm{E}}\left(\bar{\phi}_{i}\right)$ & $\mathrm{i}=1$ & $\mathrm{i}=2$ & $\mathrm{i}=3$ & $\mathrm{i}=4$ & $\mathrm{i}=5$ & $\mathrm{i}=6$ \\
\hline $\mathrm{E}=\mathrm{g} 1$ & $\mathbf{3 . 3 1 0}^{\mathbf{5}}$ & $\mathbf{1 . 3 1 0}^{\mathbf{6}}$ & $\mathbf{7 . 6 1 0}^{\mathbf{6}}$ & $3.810^{6}$ & $\mathbf{2 . 5} \mathbf{1 0}^{\mathbf{7}}$ & $\mathbf{6 . 0 1 0 ^ { 7 }}$ \\
$\mathrm{E}=\mathrm{g} 2$ & $\mathbf{1 . 4 1 0}^{\mathbf{5}}$ & $6.710^{4}$ & $9.910^{3}$ & $\mathbf{1 . 0 1 0}^{\mathbf{7}}$ & $2.010^{6}$ & $1.710^{5}$ \\
$\mathrm{E}=\mathrm{g} 3$ & $\mathbf{2 . 5 1 0}^{\mathbf{5}}$ & $\mathbf{1 . 7 1 0}^{\mathbf{6}}$ & $6.110^{5}$ & $4.710^{5}$ & $6.910^{4}$ & $1.910^{5}$ \\
\hline
\end{tabular}

The reduction process is carried out by choosing successively as experimental data Modes 6, 4 and 2 for Groups 1,2 and 3 respectively, and by assuming that these data are representative of the global behavior of the truss (coefficients $\rho_{E}$ equal to 1 ). The final results are shown in Table 3 . In this simple case, these results can be compared directly to the stiffness dispersions introduced into the deterministic model in order to simulate the experimental data: $\left[\bar{K}_{g 1}-\right.$ $\left.5 \% ; \bar{K}_{g 1}+5 \%\right],\left[\bar{K}_{g 2}-15 \% ; \bar{K}_{g 2}+5 \%\right]$ and $\left[\bar{K}_{g 3}-10 \% ; \bar{K}_{g 3}+20 \%\right]$. One can therefore note the good correspondence of the values obtained. 


\begin{tabular}{|c|c|c|c|c|}
\hline \multirow{2}{*}{ Groups } & \multirow{2}{*}{ Experimental data } & \multicolumn{3}{|c|}{ Reduced basic LOKs } \\
\cline { 3 - 5 } & & Law & {$\left[-\bar{m}_{\mathrm{E}}^{-} ; \bar{m}_{\mathrm{E}}^{+}\right]$} & Statistical moments \\
\hline$g 1$ & $\left(\Delta \omega_{6 \exp }^{2+}, \Delta \omega_{6 \text { exp }}^{2-}\right)$ & Gaussian & {$[-0.039 ; 0.041]$} & $\mu=0.001 / \sigma=0.016$ \\
$g 2$ & $\left(\Delta \omega_{4 \exp }^{2+}, \Delta \omega_{4 \exp }^{2-}\right)$ & Gaussian & {$[-0.158 ; 0.058]$} & $\mu=-0.050 / \sigma=0.042$ \\
$g 3$ & $\left(\Delta \omega_{2 \exp }^{2+}, \Delta \omega_{2 \exp }^{2-}\right)$ & uniform & {$[-0.108 ; 0.203]$} & $\mu=0.048 / \sigma=0.090$ \\
\hline
\end{tabular}

Table 3

Reduced basic LOKs

\subsection{Predictive capability}

With the basic LOKs determined above, one can calculate effective LOKs for the three remaining unused modes $(1,3$ and 5$)$ in order to evaluate the quality of the results of our reduction process. The basic LOKs are modeled using the chosen probability laws associated with the prescribed levels; then the corresponding $99 \%$ values are calculated and compared to the experimental $99 \%$ values in Table 4 . One can observe that the constraints are properly verified for Modes 1, 3 and 5, which shows that the results obtained with Modes 2, 4 and 6 are consistent.

Table 4

Comparison of eigenpulsations and eigenmodes (99\%-values) for Modes 1, 3 and 5

\begin{tabular}{|c||c|c|c|c|c||c|c|c|c|c|}
\hline$i$ & $\Delta \omega_{i \text { mod }}^{2-}$ & $\Delta \omega_{i \text { exp }}^{2-}$ & $\bar{\omega}_{i}^{2}$ & $\Delta \omega_{i \text { exp }}^{2+}$ & $\Delta \omega_{i \text { mod }}^{2+}$ & $\Delta \phi_{k i \text { mod }}^{-}$ & $\Delta \phi_{k i \text { exp }}^{-}$ & $\bar{\phi}_{k i}$ & $\Delta \phi_{k i \text { exp }}^{+}$ & $\Delta \phi_{k i \bmod }^{+}$ \\
\hline 1 & $1.3610^{6}$ & $1.3510^{6}$ & $1.4310^{6}$ & $1.5310^{6}$ & $1.5410^{6}$ & 0.85 & 0.88 & 0.95 & 0.99 & 1.01 \\
3 & $1.5810^{7}$ & $1.5810^{7}$ & $1.6410^{7}$ & $1.7110^{7}$ & $1.7010^{7}$ & -1.00 & -0.98 & -0.95 & -0.91 & -0.90 \\
5 & $5.2810^{7}$ & $5.2910^{7}$ & $5.5110^{7}$ & $5.6810^{7}$ & $5.6910^{7}$ & -0.74 & -0.72 & -0.68 & -0.62 & -0.62 \\
\hline
\end{tabular}

\section{Study of an industrial case}

\subsection{Description of the structure}

Now, let us present the application of our method to an actual industrial structure: the Sylda5 satellite support, developed by the EADS Group, which is capable of carrying two satellites simultaneously (Figure 5). Free-vibration measurements with 260 sensors were carried out by IABG on behalf of DASA/DORNIER under contract with CNES. The model proposed by EADS represents both the support itself and a cylindrical payload which simulates the presence of a satellite resting on the support. This model consists of 38 substructures made of various materials, including orthotropic sandwich materials, aluminum and steel. As the initial measurements had shown that it was absolutely essential to take into account the deformation of the ground under the support, this ground was modeled very simply using 3 torsional springs, one translational spring and a rigid-body constraint for all interface nodes between 
the ground and the support. The final model consisted of 27,648 DOFs and 9,728 elements.

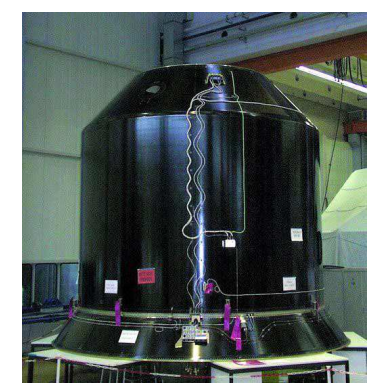

Fig. 5. Photograph of the Sylda5 support

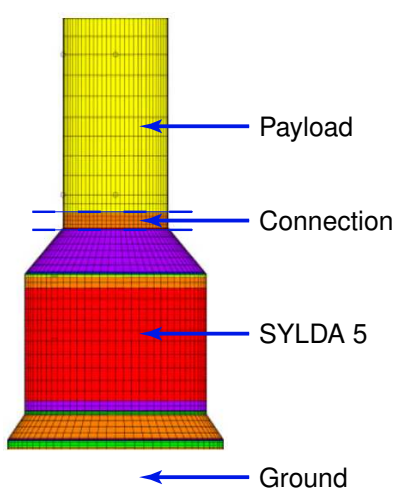

Fig. 6. The associated Sylda5 model

\subsection{Determination of the basic LOKs}

First, the model was adjusted using the method described in [2], based on the first 12 modes. The problem was then to determine the remaining LOKs. In order to do that, the structure was divided into 4 main groups of substructures, as described in Figure 6:

- Group g1 associated with the cylindrical payload;

- Group g2 containing the composite connection between the cylinder and the Sylda5 support;

- Group g3 corresponding to the Sylda5 support itself;

- Group g4 associated with the model of the ground.

Table 5

Initial LOKs

\begin{tabular}{|c|c|c|}
\hline Groups & Law being sought & $\left(\bar{m}_{\mathrm{E}}^{-0}, \bar{m}_{\mathrm{E}}^{+0}\right)$ \\
\hline $\mathrm{E}=\mathrm{g} 1$ & Gaussian & $(20 \%, 20 \%)$ \\
$\mathrm{E}=\mathrm{g} 2$ & uniform & $(20 \%, 20 \%)$ \\
$\mathrm{E}=\mathrm{g} 3$ & Gaussian & $(20 \%, 20 \%)$ \\
$\mathrm{E}=\mathrm{g} 4$ & uniform & $(50 \%, 50 \%)$ \\
\hline
\end{tabular}

Table 6 Experimental data

\begin{tabular}{|c|c|}
\hline Groups & Data used \\
\hline $\mathrm{E}=\mathrm{g} 3$ & Mode $4\left(\omega_{4 \text { exp }}^{2 \text { sup }}, \omega_{4 \text { exp }}^{2 \text { inf }}\right)$ \\
$\mathrm{E}=\mathrm{g} 1$ & Mode $8\left(\omega_{8 \text { exp }}^{2 \text { sup }}, \omega_{8 \text { exp }}^{2 \text { inf }}\right)$ \\
$\mathrm{E}=\mathrm{g} 4$ & Mode $6\left(\omega_{6 \text { exp }}^{2 \text { exp }}, \omega_{6 \text { exp }}^{2 \text { inf }}\right)$ \\
$\mathrm{E}=\mathrm{g} 2$ & Mode $3\left(\omega_{3 \text { exp }}^{2 \text { exp }}, \omega_{3 \text { exp }}^{2 \text { inf }}\right)$ \\
\hline
\end{tabular}

The reduction process was initiated by setting a priori the initial LOK levels $\left(\bar{m}_{\mathrm{E}}^{-0}, \bar{m}_{\mathrm{E}}^{+0}\right)$ (and their corresponding laws) described in Table 5. The experimental information consisted of the eigenmodes and the extrema of the eigenpulsations of a series of measurements. Table 6 indicates the order in which, and the data with which, the reduction was carried out. The results were the 
following (with $\rho_{E}=1$ ):

$$
\begin{array}{llll}
\bar{m}_{g 1}^{+}=14.4 \% & \bar{m}_{g 2}^{+}=0.0 \% & \bar{m}_{g 3}^{+}=0.0 \% & \bar{m}_{g 4}^{+}=43.5 \% \\
\bar{m}_{g 1}^{-}=0.0 \% & \bar{m}_{g 2}^{-}=6.0 \% & \bar{m}_{g 3}^{-}=1.5 \% & \bar{m}_{g 4}^{-}=0.0 \%
\end{array}
$$

These results confirm the good quality of the adjusted model of the support (g3) and of the model of the connector (both within a few \%), whereas the oversimplifications in the model of the ground resulted in a high LOK.

\section{Conclusion}

In this article, we showed some applications of the Lack-Of-Knowledge theory, which combines interval analysis with the theory of probabilities. This method enables one to quantify the uncertainties on the substructure level using quantities of interest defined over the whole structure. The reduction process presented here enables the determination of the basic LOKs for each substructure starting from a priori assumptions on their bounds. In order to do that, the experimental data are considered to be information which reduces the LOK on the structure. These investigations constitute a first step toward a general method of reduction of the LOKs.

\section{References}

[1] J. E. Mottershead, M. I. Friswell, Model updating in structural dynamics: a survey, Journal of Sound and Vibration 167 (2) (1993) 347-375.

[2] P. Ladevèze, A. Chouaki, Application of a posteriori error estimation for structural model updating, Inverse Problems 15 (1999) 49-58.

[3] G. I. Schuëller, Computational stochastic mechanics - recent advances, Computers and Structures 79 (2001) 2225-2234.

[4] Y. Ben-Haim, Information-Gap Decision Theory, Academic Press, London, 2001.

[5] G. Klir, Generalized information theory, Fuzzy Sets and Systems 40 (1991) 127-142.

[6] P. Walley, Statistical Reasoning with Imprecise Probabilities, Chapman and Hall, 1990.

[7] P. Ladevèze, On a theory of the lack of knowledge in structural computation, Technical Note SY/XS 136 127, EADS Launch Vehicles, in French (April 2002). 
[8] P. Ladevèze, G. Puel, T. Romeuf, On a strategy of reduction of the lack of knowledge (LOK) in model validation, in: Proceedings of the Fourth International Conference on Sensitivity Analysis of Modeling Output, Santa Fe, New Mexico, 2004.

[9] R. M. Moore, Interval Analysis, Prentice-Hall, 1966.

[10] R. Moore, Methods and Applications of Interval Analysis, Studies in Applied Mathematics (SIAM), 1979.

[11] P. Ladevèze, The theory of the lack of knowledge in structural dynamics: Development and first examples, Technical Note LY22 139 423, EADS Launch Vehicles, in French (May 2003).

[12] A. P. Dempster, Upper and lower probabilities generated by a random interval, Annals of Mathematical Statistics 39 (3) (1968) 957-966.

[13] G. Shafer, A Mathematical Theory of Evidence, Princeton University Press, 1976.

[14] R. Nelson, Simplified calculations of eigenvector derivatives, AIAA Journal 14 (1976) 1201-1205.

[15] D. Barthe, A. Deraemaeker, P. Ladevèze, G. Puel, On a theory of the quantification of the lack of knowledge in structural computation, in: Proceedings of the 21st International Modal Analysis Conference (IMAC-XXI), Kissimmee, Florida, 2003.

[16] A. Saltelli, S. Tarantola, F. Campolongo, M.Ratto, Sensitivity Analysis in Practice: A Guide to Assessing Scientific Models, John Wiley and Sons, 2004. 